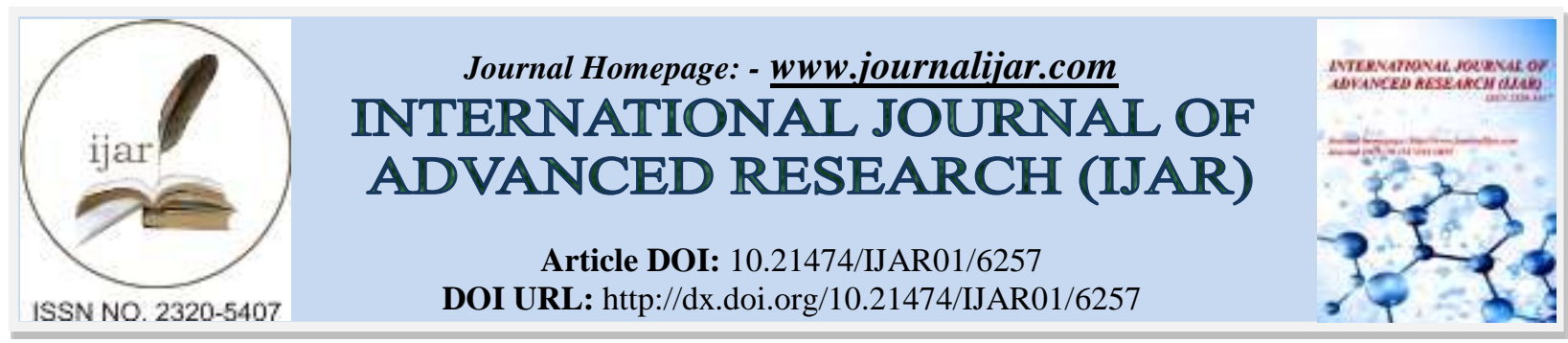

RESEARCH ARTICLE

\title{
PERCEIVED BENEFITS AND BARRIERS OF EXERCISE AMONG FEMALE UNIVERSITY STUDENTS.
}

\author{
Nanees A. Gad ${ }^{1,3}$, Mervat M. Arrab ${ }^{2,3}$ and Shmookh M. Alsayed ${ }^{3}$. \\ 1. Community Medicine Department, Faculty of Medicine, Ain Shams University, Egypt. \\ 2. Community Health Nursing, Faculty of Nursing, Menoufia University. \\ 3. Public Health department, College of Applied Medical Sciences, King Khalid University Khamis Mushait, \\ Kindom of Saudi Arabia.
}

\section{Manuscript Info}

(..........................

Manuscript History

Received: 09 November 2017

Final Accepted: 11 December 2017

Published: January 2018

Key words:-

Physical activity; benefits; barriers; university students.

\section{Abstract}

Background: Many individuals do not participate in sufficient physical activity due to low perceived benefits and high-perceived barriers to exercise.

Objectives: To assess the level of perceived benefits and barriers to physical activity, describe the level of physical activity as regards frequency of exercise and study relation between physical activity status and perceived exercise benefits and barriers among female university students in KKU.

Subjects and methods: A cross-sectional study carried out at Mahala campus for Girls, KKU. 400 students from four colleges were selected by simple random sampling. The Exercise Benefits/Barriers Scale used to determine perceived the benefits and barriers to exercise. The Godin questionnaire also used to determine frequency of exercise

Results: The benefits mean value was 3.29 , compared to the barriers mean value of 2.69, indicating strong agreement with the benefits of exercise. The most perceived benefit item was; "Exercising improves my self-concept", whilst the most perceived barrier item was; "There are too few places for me to exercise". About $65 \%$ of students were physically inactive. Inactive students had significantly higher mean barrier score than active students. Obese students and overweight had significantly higher mean barrier score than normal-weight students.

Conclusions and recommendations: The highest mean of benefits was the psychological outlook then followed by Physical performance, Life Enhancement, Preventive health, Social interreaction subscale respectively. The highest mean of barriers was the Time expenditure subscale then followed by the Exercise milieu, Physical exertion), Family discouragement subscale respectively. Applied interventions need to assist female university students to overcome their barrier to participate in physical activity.

Copy Right, IJAR, 2018,. All rights reserved. 


\section{Introduction:-}

A top priority of any country should be fitness and Physical activity. It has been approved that physical activity reduces from high school to college and most of the college students suffered a decrease in physical activity level following the graduation time (Rajappan et al, 2016). In 2010, the WHO reported that 23\% of adults aged 18 years old and above and $81 \%$ of adolescents aged $11-17$ years were insufficiently physically active Worldwide.

About 3.2 million deaths each year related to insufficient physical activity, and stated that physical activity can reduce mortality. Therefore, the WHO sets a global target by 2025 promote world population's health by reducing $10 \%$ in the prevalence of insufficient physical activity (WHO, 2011). Promoting a healthy lifestyle among university students first step is determining perceived barriers to physical activity. (Raynor et al., 2010). Regular physical activity can improve psychological health and physical fitness and prevent a variety of diseases including cardiovascular. (Sultoni \& Suherman., 2017)

A contributing factor for obesity, weight gain, coronary heart disease, and other life-threatening illnesses mainly physical inactivity (Munford, 2011). A critical period of adolescence is the transition between high school and university that physical activity level turns down during adolescence and activity behavior starts to decreased as adults (Bray, 2007).

The global mortality $4^{\text {th }}$ leading risk factor is physical inactivity and it is a contributing factor in the development of various chronic diseases such as hypertension, cancer and heart diseases causing 3.2 million deaths worldwide. The World Health Organization reports that about $60 \%$ of the global population do not meet the recommended daily minimum of physical activity (WHO, 2010).

In Saudi Arabia (KSA) The economic growth surge in recent years responsible of facing challenges to combat chronic health problems related to sedentary lifestyles, diet and other behavior changes associated with riches of the country. In addition, developments in city planning that require the use of automobiles for all trips (Al-Hazzaa et al., 2011), may be leading to physical inactivity and a low down level of physical fitness. Studies conducted in Saudi Arabia suggests a high prevalence $43.3-99.5 \%$ of physical inactivity among Saudi children and among adults (Khalaf et al., 2013). The southwestern region college students of Saudi Arabia suffered from high prevalence of physical inactivity and inactive leisure time among (Awadalla et al., 2016).

Analyzing university students 'barriers to physical activity is a cornerstone to address needs and promote physical activity among college students by health officials (Alsahli, 2016). The reasons why university students do not participate in sufficient physical activity (personal, interpersonal, environmental, and social and policy factors) are essential, perceived barriers to being active are the main predictor of health behavior change. (Lovell et al., 2010).

Aim:

The purpose of the present study was to examine the perceived benefits and barriers of physical activity among female university students to improve their physical and mental health. The specific objectives were to:

- To assess the perceived benefits and barriers to physical activity among female university students in KKU

- To describe the level of physical activity as regards frequency and duration among female university students in KKU

- To study the relationship between physical activity status and perceived exercise benefits and barriers among female university students in KKU

\section{Subjects and methods:-}

Research design:

4. A cross sectional study carried out at Mahala female campus, King Khalid University (KKU), Khamis Mushait, Kindom of Saudi Arabia.

\section{Sample and Participants:}

The target population was the female students at Mahala female campus, in KKU. These include 4 colleges: applied medical science; nursing; science \& art and community.

Sampling technique: simple random sample 
Sample size:

$\mathrm{n}=\mathrm{Z} 2 \mathrm{p} \mathrm{q} / \mathrm{E} 2$

$\mathrm{Z}$ at $95 \%$ confidence $=1.96(2)$

$\mathrm{P}$ : estimated percent in population $=50 \%$

$\mathrm{q}: 100-\mathrm{p}=100-50=50 \%$

e : accepted sample errors $(0.05)$

Power $=0.8$

The total is 400

Tools:

A self-administered Questionnaire: include

A. Socio demographic characteristics (age, name of college, education, occupation of father and mother and family income)

B. Data about usual exercise habits and their frequencies based on Godin Leisure Time Exercise Questionnaire This is a brief query of usual exercise habits and their frequencies

C. Exercise benefits/barriers scale (EBBS)

According to the EBBS questionnaire (Sechrist et al, 1987), this instrument previously assessed as a reliable measure to evaluate the perceived benefits and barriers to exercise.

The EBBS questionnaire is made up of a total of 43 questions, 29 of which comprise the benefits components and 14 of which make up the barrier components.

The benefits component are further divided in five subscales: life enhancement ( 8 questions), physical performance (8 questions), psychological outlook (6 questions), social interaction (4 questions), and preventative health (3 questions).

The barriers component is categorized as four subscales: exercise milieu (6 questions), time expenditure (3 questions), physical exertion (3 questions), and family discouragement (2 questions).

The Likert 4-point scoring system was used to score all items in the benefits and barriers scale; whereby $4=$ 'strongly agree'; 3 = 'agree'; $2=$ 'disagree'; and $1=$ 'strongly disagree'.

\section{Procedure:}

Approval to conduct this study was obtained from Dean of applied medical science, nursing; science \& art and community colleges, King Khalid University. Analysis of the findings carried out by using SPSS software package version 20 .

To assess the perceived benefits and barriers to physical activity: A descriptive analysis performed. Possible scores of Likert scale ranged from one to four; four represented the highest perception of benefit and perception of barrier. The scores were computed for both the total benefits, total barriers and total score. Scores on the total instrument can range from 43 to 172 . The higher the score, the more positively the individual perceives exercise. The score range of benefits is between 29 and 116 and score range of barrier is ranged between 14 and 56. The means of the individual EBBS items as well as the mean total of all benefits and barriers items was calculated. For each scale and subscale, the items were added then divided by number of items included in that scale to calculate the mean score.

To describe the level of physical activity status. The physical activity status was classified according to Godin Leisure Time Exercise Questionnaire Scoring for health contribution. Weekly frequencies of strenuous and moderate, activities are multiplied by 9 and 5, respectively. Total weekly leisure activity was calculated in by summing the products of the separate components 1- 24 units or more: Active, 2- 23 units or less: Insufficiently active. This cut point ( 24 units or more) is more near of the «basic» public health recommendations (Minimal weekly volume with strenuous and/or moderate physical activity).

To study the relation between physical activity status and perceived exercise benefits and barriers by using independent $\mathrm{t}$ test.

To study the relation between BMI groups and perceived exercise benefits and barriers, ANOVA test was used. 


\section{Results:-}

Table (1) demonstrates Socio-demographic characteristics of the study sample. It was clear that, 97.3\% of females university students were Saudi, the majority of student's residence $84.3 \%$ were urban. Slightly more than one third of students were married.

Table (2) and Figure (1) show the exercise benefits scale: mean and standard deviation of each item. It was clear that female's university students either agreed or strongly agreed with most of the benefits items as regards regular exercise

- $\quad$ Under the life enhancement subscale, the item (32-. Exercising improves my self-concept had the highest mean (3.48).

- Under the physical performance subscale, the item (43- Exercise improves the way my body looks had the highest mean (3.45), followed by (15- Exercising increases my level of physical fitness mean (3.42).

- Under the psychological outlook subscale, the item (1- I enjoy exercise had highest mean (3.45) followed by item 3- Exercise improves my mental health had mean (3.40).

- Under Social interreaction subscale, the item (38- Exercise is good entertainment for me had highest mean (3.26)

- Under Preventive health subscale, the item (13- Exercising will keep me from having high blood pressure) had the highest mean (3.26)

- The highest mean was of the psychological outlook (3.35) then followed by Physical performance (3.34) Life Enhancement subscale (3.30), Preventive health subscale (3.23) and Social interreaction subscale (3.12) respectively.

Table (3) and Figure (2) show the exercise barriers scale: mean and standard deviation of each item.

This table and figure revealed that females' university students agreed with many of the barriers items

Under the Exercise milieu subscale, the item (9- Places for me to exercise are too far away had the highest mean (2.95).

-Under the Exercise milieu subscale, the item (16- Exercise facilities do not have convenient schedules for me had the highest mean (2.97).

-Under the Exercise milieu subscale, the item (42- There are too few places for me to exercise had the highest mean (3.20).

-Under the Time expenditure subscale, the item (4- Exercising takes too much of my time had the highest mean (2.84).

-Under the Time expenditure subscale, the item (37- Exercise takes too much time from my family responsibilities had the highest mean (2.80).

The highest mean was the Time expenditure subscale (2.80) then followed by the Exercise milieu subscale (2.70), Physical exertion subscale (2.63), and Family discouragement subscale (2.55).

Table (4) shows the mean benefit, barrier and total score among study sample. It shows that the mean benefit score was 3.29, while mean barrier score was 2.69 .

Table (5) and figure (3) show Comparison between mean benefit score and mean barrier score. Which revealed that there is significant difference between mean benefit score and mean barrier score.

Figure 4 shows places where students practice sports. This figure revealed that $47 \%$ of students practice sports at their home while $22.5 \%$ practice sport at Gym.

Figure 5 presents Students physical activity. It was clear that about $35 \%$ of students were active, While $65 \%$ were inactive.

Table (6) shows the frequency of physical exercise practice among the study sample. It was obvious that, slightly more than half (57.3) of students did not practice heavy activity, about (30.3) of student did not practice Moerate activity, about (43.0) of students walk every day about $15 \mathrm{~min}$.

The relation between Activity behaviour and socio-demographic factors: The mother and father's level of education, the students' marital status and residence all had no significant impact on the students' physical activity. 
Table (7) shows the relation between activity status and benefits and barrier scores. The table shows that, Inactive students had higher score than active students as regards barriers scores and the difference was significant.

Table (8) shows relation between BMI groups and benefits and barrier scores. It was clear that, obese students and overweight had higher mean barrier score than normal weight students and the difference was statistically significant.

Table (9) shows the relation between activity status and benefits subscales score.

It was obvious that, active students had higher mean social subscale than inactive students did. The difference was statistically significant.

Table (10) shows the relation between activity status and barriers subscales score. It was clear that inactive students had higher mean score as regards all barriers subscales except Mean time expenditure score. The difference was statistically significant.

Table 1:- Socio-demographic characteristics of the study sample

\begin{tabular}{|c|c|c|c|}
\hline & & Frequency & Percent \\
\hline \multirow[t]{3}{*}{ Race } & 1- Saudi & 389 & 97.3 \\
\hline & 2- non-Saudi & 11 & 2.8 \\
\hline & Total & 400 & 100.0 \\
\hline \multirow[t]{3}{*}{ Residence } & 1- urban & 337 & 84.3 \\
\hline & 2- rural & 63 & 15.8 \\
\hline & Total & 400 & 100.0 \\
\hline \multirow[t]{3}{*}{ Marital status } & 1- married & 146 & 36.5 \\
\hline & 2- single & 254 & 63.5 \\
\hline & Total & 400 & 100.0 \\
\hline \multirow[t]{5}{*}{ Father education } & 1- uneducated & 24 & 6.0 \\
\hline & 2- primary education & 35 & 8.8 \\
\hline & 3- intermediate & 117 & 29.3 \\
\hline & 4- university education & 224 & 56.0 \\
\hline & Total & 400 & 100.0 \\
\hline \multirow[t]{5}{*}{ Mother education } & 1- uneducated & 58 & 14.5 \\
\hline & 2- primary education & 63 & 15.8 \\
\hline & 3- intermediate & 104 & 26.0 \\
\hline & 4- university education & 175 & 43.8 \\
\hline & Total & 400 & 100.0 \\
\hline
\end{tabular}

Table 2:- The exercise benefits scale: mean and standard deviation of each item

\begin{tabular}{|l|l|l|}
\hline Life Enhancement subscale & Mean & SD \\
\hline 25- My disposition is improved with exercise & 3.22 & .905 \\
\hline 26.Exercising helps me sleep better at night & 3.31 & .800 \\
\hline 39. Exercise helps me decrease fatigue. & 3.17 & .896 \\
\hline 32. Exercising improves my self-concept. & $\mathbf{3 . 4 8}$ & $\mathbf{. 7 7 8}$ \\
\hline 34. Exercising increases my mental alertness. & 3.32 & .787 \\
\hline 35. Exercise allows me to carry out normal activities without tired. & 3.29 & .840 \\
\hline 36. Exercise improves the quality of my work. & 3.29 & .835 \\
\hline 41. Exercise improves overall body functioning for me. & 3.35 & .820 \\
\hline Mean total Life Enhancement subscale & $\mathbf{3 . 3 0}$ & $\mathbf{. 5 5 8}$ \\
\hline Physical performance subscale & $\mathbf{M e a n}$ & SD \\
\hline 7- Exercise increases my muscle strength & 3.31 & .865 \\
\hline 15- Exercising increases my level of physical fitness & $\mathbf{3 . 4 2}$ & $\mathbf{. 7 7 4}$ \\
\hline 17- My muscle tone is improved with exercise. & 3.34 & .812 \\
\hline 18- Exercising improves functioning of my CVS. & 3.38 & .775 \\
\hline 22- Exercise increases my stamina & 3.23 \\
\hline
\end{tabular}




\begin{tabular}{|l|l|l|}
\hline 23- Exercise improves my flexibility & 3.29 & .827 \\
\hline 31- My physical endurance is improved by exercising. & 3.31 & .842 \\
\hline 43- Exercise improves the way my body looks. & $\mathbf{3 . 4 5}$ & $\mathbf{. 8 5 9}$ \\
\hline & $\mathbf{3 . 3 4}$ & $\mathbf{. 5 5 6}$ \\
\hline Psychological outlook subscale & $\mathbf{M e a n}$ & $\mathbf{S D}$ \\
\hline 1- I enjoy exercise. & $\mathbf{3 . 4 5}$ & $\mathbf{. 7 6 7}$ \\
\hline 2- Exercise decreases feelings of stress for me. & 3.34 & .773 \\
\hline 3- Exercise improves my mental health. & $\mathbf{3 . 4 0}$ & $\mathbf{. 7 4 2}$ \\
\hline 8- Exercise gives me a sense of personal accomplishment & 3.32 & .825 \\
\hline 10- Exercising makes me feel relaxed. & 3.28 & .852 \\
\hline 20- I have improved feelings of wellbeing from exercise. & 3.26 & .866 \\
\hline & $\mathbf{3 . 3 5}$ & $\mathbf{. 5 5 3}$ \\
\hline Social inter reaction subscale & $\mathbf{M e a n}$ & $\mathbf{S D}$ \\
\hline 11- Exercising lets me have contact with friends I enjoy. & 3.02 & 1.01 \\
\hline 30- Exercising is a good way for me to meet new people & 3.07 & .979 \\
\hline 38- Exercise is good entertainment for me. & 3.26 & .828 \\
\hline 39- Exercising increases my acceptance by others & 3.11 & .912 \\
\hline & $\mathbf{3 . 1 2}$ & $\mathbf{. 6 5 8}$ \\
\hline Preventive health subscale & $\mathbf{M e a n}$ & $\mathbf{S D}$ \\
\hline 5- I will prevent heart attacks by exercising & 3.25 & .844 \\
\hline 13- Exercising will keep me from having high blood pressure & 3.31 & .779 \\
\hline 27- I will live longer if I exercise & 3.10 & .927 \\
\hline & $\mathbf{3 . 2 3}$ & $\mathbf{. 6 4 2}$ \\
\hline
\end{tabular}
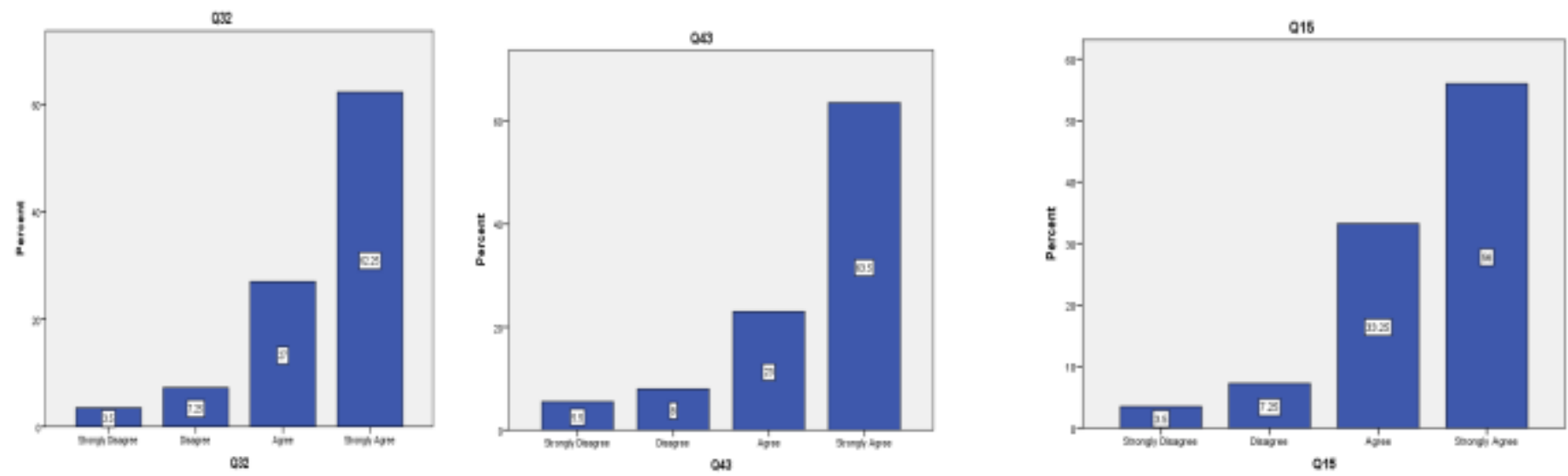

(Fig.1.A)Exercising improves my self-concept. (Fig.1.B) Exercise improves the way my body looks. (Fig.1.C) Exercising increases my level of physical fitness

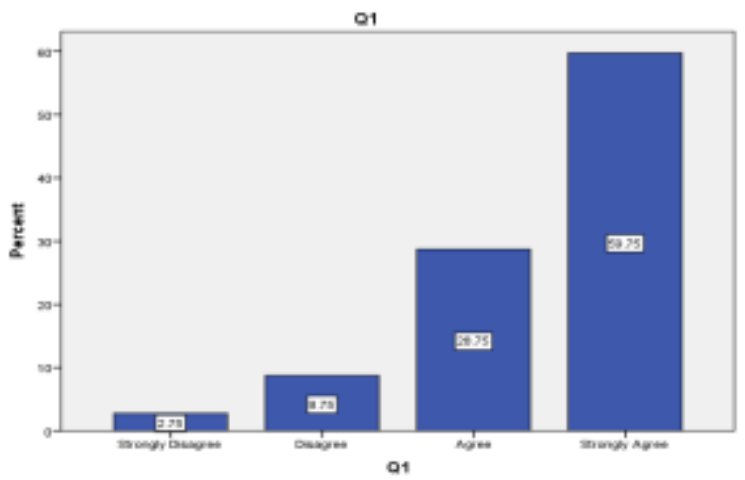

(Fig.1.D) I enjoy exercise

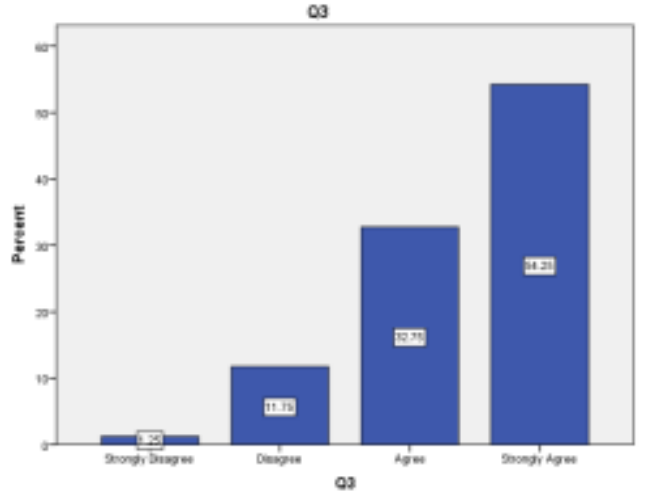

(Fig.1.E) Exercise improves my mental health 
Table 3:- The exercise barriers scale: mean and standard deviation of each item

\begin{tabular}{|c|c|c|}
\hline Exercise milieu subscale & Mean & SD \\
\hline 9- Places for me to exercise are too far away & 2.95 & 1.01 \\
\hline 12- I am too embarrassed to exercise. & 2.29 & 1.04 \\
\hline 14- It costs too much to exercise. & 2.64 & 1.04 \\
\hline 16- Exercise facilities do not have convenient schedules for me. & 2.97 & .942 \\
\hline 28- I think people in exercise clothes look funny. & 2.17 & 1.10 \\
\hline \multirow[t]{2}{*}{ 42- There are too few places for me to exercise } & 3.20 & .936 \\
\hline & 2.70 & .639 \\
\hline Time expenditure subscale & Mean & SD \\
\hline 4- Exercising takes too much of my time & 2.84 & .908 \\
\hline 24- Exercise takes too much time from family relationships & 2.79 & .948 \\
\hline \multirow[t]{2}{*}{ 37- Exercise takes too much time from my family responsibilities } & 2.80 & .989 \\
\hline & 2.80 & .724 \\
\hline Physical exertion subscale & Mean & SD \\
\hline 6- Exercise tires me. & 2.65 & .923 \\
\hline 19- I am fatigued by exercise & 2.67 & .941 \\
\hline \multirow[t]{2}{*}{ 40- Exercise is hard work for me } & 2.55 & 1.06 \\
\hline & 2.63 & .730 \\
\hline Family discouragement subscale & Mean & SD \\
\hline 21- My spouse (or significant other) does not encourage exercising & 2.65 & 1.04 \\
\hline \multirow[t]{2}{*}{ 33- My family members do not encourage me to exercise } & 2.46 & 1.02 \\
\hline & 2.55 & .867 \\
\hline
\end{tabular}

Places for me to excreise tro toe far away

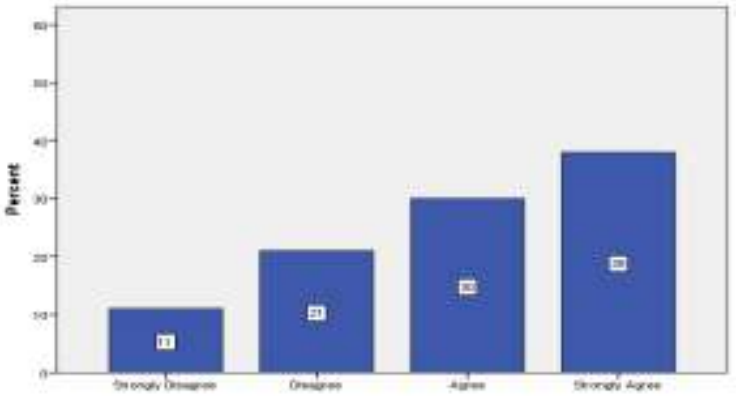

(Fig.2.A)

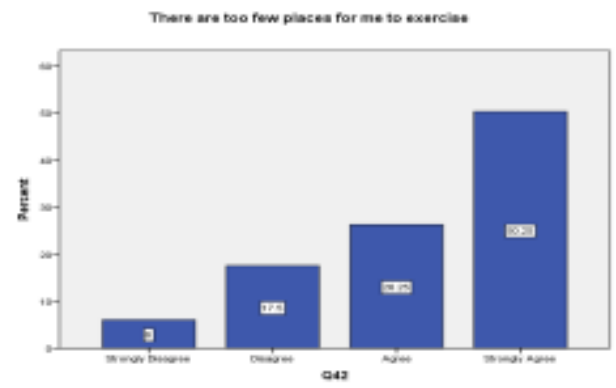

(Fig.2.C)
Exeroise facilities de not have oonvenient sehedules for me.

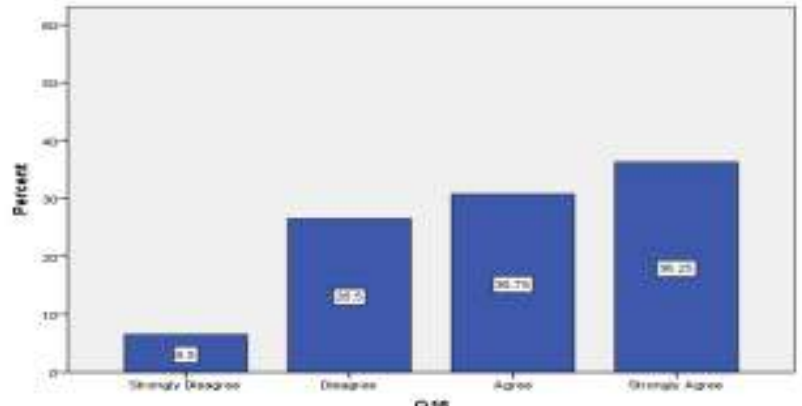

(Fig.2.B)

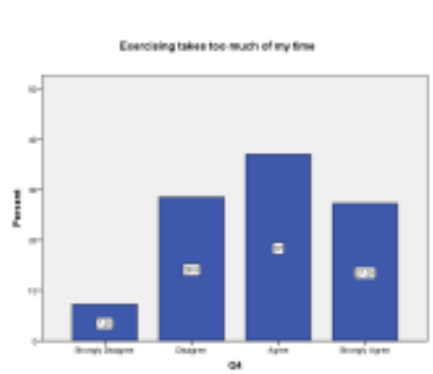

(Fig.2.D)

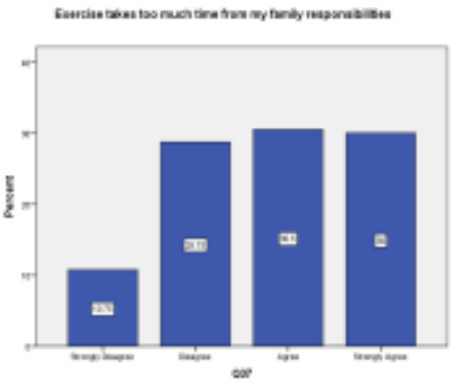

(Fig.2.E) 
Table 4:- Mean benefit, barrier and total score among study sample

\begin{tabular}{|l|l|l|l|l|}
\hline & Min & Max & Mean & Std. Deviation \\
\hline Barriers score & 14.0 & 56.0 & 37.66 & 8.26 \\
\hline Mean barrier score & 1.0 & 4.0 & $\mathbf{2 . 6 9}$ & 0.59 \\
\hline Benefits score & 36.0 & 116.0 & 95.38 & 14.48 \\
\hline Mean benefits score & 1.24 & 4 & $\mathbf{3 . 2 9}$ & 0.49 \\
\hline Total score & 50.0 & 172.0 & 133.04 & 17.86 \\
\hline Mean total score & 1.16 & 4.0 & 3.09 & 0.42 \\
\hline
\end{tabular}

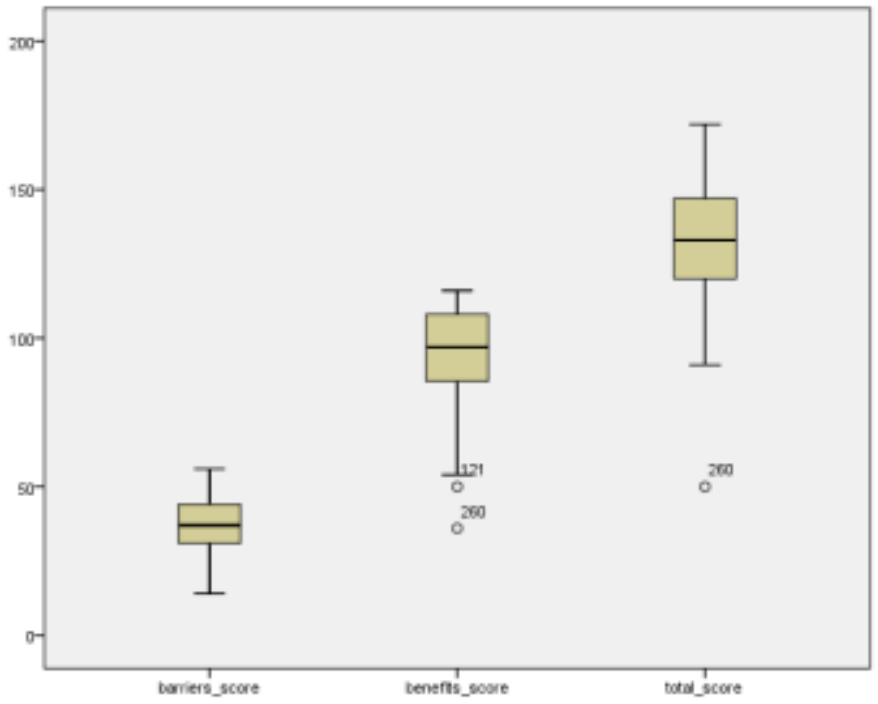

Figure 3:- Comparison between mean benefit score and mean barrier score.

Table 5:- Comparison between mean benefit score and mean barrier score

\begin{tabular}{|l|l|l|l|}
\hline \multicolumn{2}{|l|}{} & Mean & Std. Deviation \\
\hline \multirow{2}{|l|}{ Mean benefits score } & 3.2892 & .49935 \\
\cline { 2 - 4 } & Mean barriers score & 2.6900 & .59031 \\
\hline \multicolumn{2}{|l|}{} & Paired t test 17.01 & $\mathrm{P}=0.000$ \\
\hline
\end{tabular}

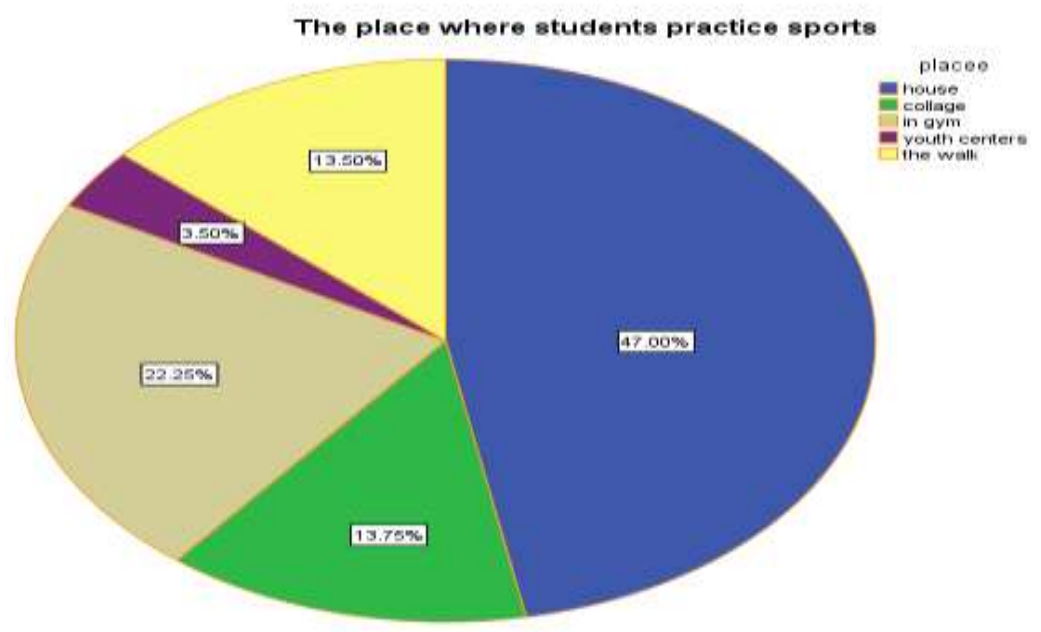

Figure 4:- Places where students practice sports. 


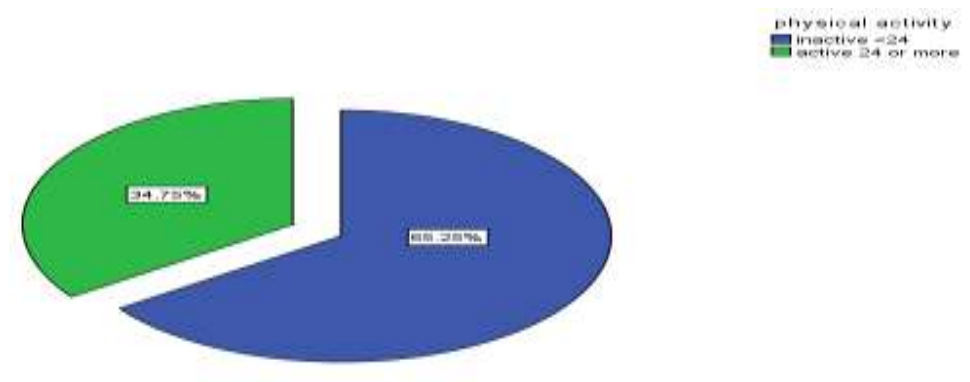

Figure 5:- Students physical activity.

Table 6:- Frequency of physical exercise practice among the study sample.

\begin{tabular}{|c|c|c|c|c|c|c|c|c|c|}
\hline & \multicolumn{9}{|c|}{ Frequency of physical exercise practice } \\
\hline & Never & $\begin{array}{l}\text { once a } \\
\text { week }\end{array}$ & $\begin{array}{l}\text { two } \\
\text { times a } \\
\text { week }\end{array}$ & $\begin{array}{l}\text { three } \\
\text { times a } \\
\text { week }\end{array}$ & $\begin{array}{l}\text { four } \\
\text { times a } \\
\text { week }\end{array}$ & $\begin{array}{l}\text { five } \\
\text { times a } \\
\text { week }\end{array}$ & $\begin{array}{l}\text { six } \\
\text { times a } \\
\text { week }\end{array}$ & $\begin{array}{l}\text { seven } \\
\text { times a } \\
\text { week }\end{array}$ & Total \\
\hline $\begin{array}{l}\text { Heavy } \\
\text { Frequency } \\
\text { Percent }\end{array}$ & $\begin{array}{l}229 \\
57.3 \\
\end{array}$ & $\begin{array}{l}59 \\
14.8 \\
\end{array}$ & $\begin{array}{l}49 \\
12.3 \\
\end{array}$ & $\begin{array}{l}34 \\
8.5 \\
\end{array}$ & $\begin{array}{l}18 \\
4.5 \\
\end{array}$ & $\begin{array}{l}6 \\
1.5 \\
\end{array}$ & $\begin{array}{l}1 \\
.3 \\
\end{array}$ & $\begin{array}{l}4 \\
1.0 \\
\end{array}$ & $\begin{array}{l}400 \\
100.0\end{array}$ \\
\hline $\begin{array}{l}\text { Moderate } \\
\text { Frequency } \\
\text { Percent }\end{array}$ & $\begin{array}{l}121 \\
30.3\end{array}$ & $\begin{array}{l}59 \\
14.8\end{array}$ & $\begin{array}{l}74 \\
18.5\end{array}$ & $\begin{array}{l}48 \\
12.0\end{array}$ & $\begin{array}{l}29 \\
7.3\end{array}$ & $\begin{array}{l}26 \\
6.5\end{array}$ & $\begin{array}{l}36 \\
9.0\end{array}$ & $\begin{array}{l}7 \\
1.8\end{array}$ & $\begin{array}{l}400 \\
100.0\end{array}$ \\
\hline $\begin{array}{l}\text { walk about } \\
\mathbf{1 5} \text { min } \\
\text { Frequency } \\
\text { Percent }\end{array}$ & $\begin{array}{l}15 \\
3.8\end{array}$ & $\begin{array}{l}26 \\
6.5\end{array}$ & $\begin{array}{l}22 \\
5.5\end{array}$ & $\begin{array}{l}45 \\
11.3\end{array}$ & $\begin{array}{l}49 \\
12.3\end{array}$ & $\begin{array}{l}42 \\
10.5\end{array}$ & $\begin{array}{l}29 \\
7.3\end{array}$ & $\begin{array}{l}172 \\
43.0\end{array}$ & $\begin{array}{l}400 \\
100.0\end{array}$ \\
\hline
\end{tabular}

Table 7:- Relation between activity status and benefits and barrier scores

\begin{tabular}{|l|l|l|l|l|l|}
\hline & Activity status & $\mathrm{N}$ & Mean & Std. Deviation & P value \\
\hline \multirow{2}{*}{ barriers score } & inactive $<24$ & 261 & 38.5364 & 8.40991 & $<0.05$ \\
\cline { 2 - 5 } & active 24 or more & 139 & 36.0144 & 7.74876 & \\
\hline \multirow{2}{*}{ benefits score } & inactive <24 & 261 & 95.8276 & 13.96303 & $>0.05$ \\
\cline { 2 - 5 } & active 24 or more & 139 & 94.5612 & 15.42495 & \\
\hline
\end{tabular}

Table 8:- Relation between BMI groups and benefits and barrier scores

\begin{tabular}{|c|c|c|c|c|}
\hline & & Number & Mean & SD \\
\hline \multirow{7}{*}{ barriers score } & underweight & 35 & 34.65 & 8.42 \\
\hline & normal & 245 & 37.15 & 8.22 \\
\hline & overweight & 75 & 39.33 & 8.41 \\
\hline & obese I & 27 & 40.44 & 7.21 \\
\hline & obese II & 6 & 42.50 & 4.67 \\
\hline & Total & 388 & 37.65 & 8.28 \\
\hline & & \multicolumn{3}{|c|}{ F test $3.506 \quad P<0.05$} \\
\hline \multirow{7}{*}{ benefits score } & underweight & 35 & 94.45 & 12.84 \\
\hline & normal & 245 & 95.51 & 14.85 \\
\hline & overweight & 75 & 96.32 & 13.63 \\
\hline & obese I & 27 & 91.40 & 15.00 \\
\hline & obese II & 6 & 104.50 & 10.87 \\
\hline & Total & 388 & 95.42 & 14.44 \\
\hline & & \multicolumn{3}{|c|}{\begin{tabular}{|lll} 
F test & 1.23 & $\mathrm{P}>0.05$
\end{tabular}} \\
\hline
\end{tabular}


Table 9:- Relation between activity status and benefits subscales score

\begin{tabular}{|c|c|c|c|c|c|}
\hline & Activity status & $\mathrm{N}$ & Mean & Std. Deviation & Std. Error Mean \\
\hline \multirow{2}{*}{$\begin{array}{l}\text { Mean life enhancement } \\
\text { score }\end{array}$} & inactive $<24$ & 261 & 3.3209 & .54520 & .03375 \\
\hline & active 24 or more & 139 & 3.2725 & .58228 & .04939 \\
\hline & & \multicolumn{4}{|c|}{$\mathrm{P}>0.05$} \\
\hline \multirow{3}{*}{$\begin{array}{l}\text { Mean physical } \\
\text { performance score }\end{array}$} & inactive $<24$ & 261 & 3.3764 & .52914 & .03275 \\
\hline & active 24 or more & 139 & 3.2716 & .59965 & .05086 \\
\hline & & \multicolumn{4}{|c|}{$\mathrm{P}>0.05$} \\
\hline \multirow{2}{*}{$\begin{array}{l}\text { Mean Psychological } \\
\text { Outlook score }\end{array}$} & inactive $<24$ & 261 & 3.37 & 0.03 & \\
\hline & active 24 or more & 139 & 3.28 & 0.05 & \\
\hline \multirow{3}{*}{ Mean social score } & inactive $<24$ & 261 & 3.0690 & .66689 & .04128 \\
\hline & active 24 or more & 139 & 3.2122 & .63340 & .05372 \\
\hline & & \multicolumn{4}{|c|}{$\mathrm{P}<0.05$} \\
\hline \multirow{3}{*}{ Mean preventive score } & inactive $<24$ & 261 & 3.2337 & .61279 & .03793 \\
\hline & active 24 or more & 139 & 3.2134 & .69737 & .05915 \\
\hline & & \multicolumn{4}{|c|}{$\mathrm{P}>0.05$} \\
\hline
\end{tabular}

Table 10:- Relation between activity status and barriers subscales score

\begin{tabular}{|c|c|c|c|c|}
\hline & Activity status & $\mathrm{N}$ & Mean & Std. Deviation \\
\hline \multirow{2}{*}{ Mean exercise milieu score } & inactive $<24$ & 261 & 2.7612 & .63985 \\
\hline & active 24 or more & 139 & 2.5983 & .62741 \\
\hline & & \multicolumn{3}{|c|}{$\begin{array}{ll}\text { T test } 2.440 & \mathrm{P} .015 \\
\end{array}$} \\
\hline \multirow{3}{*}{$\begin{array}{l}\text { Mean time } \\
\text { expenditure score }\end{array}$} & inactive $<24$ & 261 & 2.8519 & .72160 \\
\hline & active 24 or more & 139 & 2.7290 & .72628 \\
\hline & & \multicolumn{3}{|c|}{ T test $1.618 \quad \mathrm{P} .107$} \\
\hline \multirow{3}{*}{$\begin{array}{r}\text { Mean physical } \\
\text { exertion score }\end{array}$} & inactive $<24$ & 261 & 2.7229 & .72341 \\
\hline & active 24 or more & 139 & 2.4556 & .71463 \\
\hline & & 3.54 & $\mathrm{P}$. & \\
\hline \multirow{3}{*}{$\begin{array}{l}\text { Mean family } \\
\text { Discouragement score }\end{array}$} & inactive $<24$ & 261 & 2.6226 & .87173 \\
\hline & active 24 or more & 139 & 2.4353 & .84877 \\
\hline & & \multicolumn{3}{|c|}{$\begin{array}{lll}2.082 & \mathrm{P} & .038\end{array}$} \\
\hline
\end{tabular}

\section{Discussion:-}

A university setting is vital in promoting health-enhancing behaviours. This age group is thought to be flexible and can be readily influenced to make positive changes. This is also a time whereby individuals can set habits, which then persist into adult life. (Dalibalta \& Davison, 2016)

For the exercise benefits in this work, either female university students 'agreed' or 'strongly agreed' with most of the benefits items, with a total benefit mean value was 3.29. The mean scores of subscale items were greater than 3 , indicating that the majority of our sample were perceived as benefits. While they were being neutral or nearly with an agreement with many of the barriers items, with a total barrier mean value was 2.69. Mean scores for all four barriers subscales were between 2 and 3 which we interpreted to mean neutral. The perceived benefits among studied sample were significantly greater than the perceived barriers to exercise.

In this work, the highest mean was of the psychological outlook (3.35) then followed by Physical performance (3.34) Life Enhancement subscale (3.30), Preventive health subscale (3.23) and Social inter reaction subscale (3.12) respectively.. These findings in concurrence with the results of (Dalibalta \& Davison, 2016), where they reported that the physical performance subscale had the greatest mean score of 3.39 , followed by the preventative health subscale with a mean score of 3.26 then the psychological health subscale with a mean score of 3.17. However, our findings not consistent with the results of (Dalibalta \& Davison , 2016), where they reported that their study sample had mean score in life enhancement subscale 3.04 and mean social interaction subscale score was 2.59. 
The current study revealed that regarding Life Enhancement subscale students demonstrated that 'Exercising improves my self-concept had highest (mean 3.48). However, regarding Physical performance subscale, Exercising increases my level of physical fitness had highest mean 3.42. In addition, mean score was (3.45) for Exercise improves the way, my body looks. Regarding Psychological outlook subscale, enjoy exercise had highest mean (3.45) and Exercise improves my mental health had highest mean (3.40). Exercise is good entertainment for me had highest mean 3.26 in Social interreaction subscale. Although for Preventive health subscale, Exercising will keep me from having high blood pressure mean was 3.31 .

Additionally this finding came in agreement with (Dalibalta \& Davison, 2016) who studied female university Saudi students' barriers of and benefits to participation in physical activity. (USA Middle Tennessee State University and KSA University of Hafr Al Batin), demonstrated that exercising improves my self-concept in KSA had 3.27 and in the USA had 3.28. Exercising increases my level of physical fitness KSA had 3.61, the USA had 3.42. Exercising improves the way my body looks KSA had 3.6, USA had 3.63. I enjoy exercise KSA had 3.37, USA had 3.26. Exercise improves my mental health KSA had 3.25, USA had 3.41. Moreover, the present study findings was supported by a study that was carried out by (Lovell et al., 2010) in the United Kingdom who stated that Exercising improves my self-concept 3.02. Exercising increases my level of physical fitness 3.45. Exercising improves the way my body looks 3.34. I enjoy exercise 3.05. Exercise improves my mental health 3.03.

Our results suggested that the female's university students agreed with the perceptions that exercise can improve psychological wellbeing, physical fitness and physical appearance and provide preventative health benefits. Our student sample perceived higher benefits from exercise associated with life enhancement and social interaction, with mean subscale values of 3.30 and 3.12 respectively. These in opposition with previous research on university students in the United Kingdom (UK) by (Lovell et al, 2010). and in UAE by (Dalibalta \& Davison, 2016), who reported that their sample perceived fewer benefits from exercise associated with these factors. This can be explained by in Saudi Arabia, female students perceived that exercise give them great opportunities to interact socially

Regarding the exercise barriers among studied students. The current study revealed that for the Exercise milieu subscale, There are too few places for me to exercise had the highest mean (3.20) followed by Places for me to exercise are too far away had mean (2.95) and mean (2.97) was exercise facilities do not have convenient schedules for me. However, regarding to the Time expenditure subscale Exercising takes too much of my time had the highest mean (2.84), followed by Exercise takes too much time from my family responsibilities 2.80. Regarding Physical exertion subscale, I am fatigued by exercise mean was 2.67. In addition, Family discouragement subscale my spouse (or significant other) does not encourage exercising mean score was 2.65. Moreover the present study findings were supported by a study that was carried out by the present study findings was supported by a study that was carried out by (Lovell et al,. 2010) in the United Kingdom who stated that the Exercise milieu subscale, Places for me to exercise are too far away had highest mean 2.69. Time expenditure subscale Exercising takes too much of my time had the highest mean (2.31).

This finding came in agreement with another study in Kuwaiti university students (Alsahli, 2016) who studied Female university students perceived barriers "lack of motivation", " lack of energy", "pain when I exercise", and "how " I see my body" significantly greater than male university students . In addition, revealed that "Lack of motivation" is significant barrier of females because they are not encouraged to engage in physical activity due to Kuwaiti society. Females cited more barriers to physical activity than males and overall had more internal and external barriers. The most commonly cited internal barriers to physical activity were "lack of motivation", "lack or low physical power", and "fear of injury". The most commonly cited external barriers were "lack of time", "unsuitable weather", and "lack of access to opportunities such as nearby facilities".

Another study on (Daskapan et al, 2006) at Turkish university students revealed that The total score of the external barriers was significantly higher than the score of the internal barriers Lack of time was the most important external barrier. Lack of energy was the most important internal barrier. "Lack of time due to responsibilities related to the family and social environment" and "I have never any energy to able to do any exercise". In Spanish University Students, (Lemos et al,. 2014) stated that the higher scores corresponded to "too much work"; "laziness"; "place for exercise are too far away", and "lack of time for exercise". Self-perceived health was associated with barriers to PA. 
Study on Kuwaiti students, "not having time to be physically active", was one of the main barriers reported (Musaiger et al, 2014). Furthermore, a survey on Saudi adolescents showed that lack of time was the primary reason for inactivity for both males and females (Al-Hazzaa et al, 2014).

(Alsahli M, 2016) cited barriers for both the USA and KSA university groups were "I am too embarrassed to exercise" and "I think people in exercise clothes look funny". The strongest endorsement from both the KSA and USA participants, reflected by a mean score > 3.6, was for the item: Exercise improves the way my body looks. This finding was consistent with the last surveys, among undergraduate students in the USA and UK, that suggesting the most important perceived benefits were concerned with physical appearance (Lovell, et al., 2010). It was also consistent with those reported by Samara, et al. (2015) conducted with female students in the KSA, which revealed that participants had good knowledge of the benefits of physical activity. (Alsahli M, 2016) concluded that female Saudi students attend universities in the KSA or USA, they both appear to similarly perceive the strength of their Exercise Benefits to physical activity. The reason is cultural, females tend to be less social with strangers, especially in public areas. For example, Saudi females in sports clubs are less likely to approach and engage in conversation with other females they do not know.

Regarding Mean benefit, barrier and total score among study sample, the current study revealed that higher total mean benefits score 3.29 compared to a total Mean barrier score 2.69. The current study finding was consistent with (Dalibalta \& Davison , 2016) who demonstrated that university students perceive higher levels of benefits than barriers to exercise with a total benefits mean value of 3.09, compared to a total barriers mean value of 2.16.

Regarding physical activity among studied students. The current study revealed that about $35 \%$ of students were active, While $65 \%$ were inactive this means that the benefit/barrier ratio of 1.22 was not enough to motivate these students to be active. The present study findings were supported by a study that was carried out by (Daskapan et al, 2006) at Turkish university students who stated that the prevalence of physical inactivity increased in the 15-18year-old age group in both sexes.

\section{Conclusions:-}

The female university students perceive higher levels of benefits than barriers to exercise. The highest mean of benefits was the psychological outlook then followed by Physical performance, Life Enhancement, Preventive health, Social interreaction subscale respectively. The highest mean of barriers was the Time expenditure subscale then followed by the Exercise milieu, Physical exertion), Family discouragement subscale respectively. Applied interventions need to assist female university students to overcome their barrier to participate in physical activity.

\section{Recommendations:-}

For future studies, young population provision of good physical activity health promotion program early in life to overcome any perceived barriers and to avoid future risk of chronic disease. Appropriate places should be available for females to encourage them to participate in physical activity, a larger number of public facilities for sports and exercise should established specifically for women and adolescent girls.

\section{Acknowledgments:-}

We would like to acknowledge our students for assistance in data collection (Bashayer Abdullah alahmari and Mashael alasmari) 


\section{References:-}

1. Al-Hazzaa, H.M., Abahussain, N.A., Al-Sobayel, H.I., Qahwaji, D.M. \& Musaiger, A.O. (2011): Physical activity, sedentary behaviors and dietary habits among Saudi adolescents relative to age, gender and region. Int J Behav Nutr Phys Act., 8:140. PMID:22188825

2. Al-Hazzaa, H., Alahmadi, M., Al-Sobayel, H., Abahussain, N., Qahwaji, D. \& Musaiger, A. (2014): Patterns and determinants of physical activity among Saudi adolescents. J Phys Act Health. 11(6):1202-11.

3. Alsahli M. S. (2016): Benefits and barriers to physical activity among Saudi female university students in the kingdom of Saudi Arabia and the United States. Master of Science in health and human performance. Middle Tennessee State University. Published by ProQuest LLC.

4. Alsahli, S.M. (2016): Barriers to physical activity among Kuwaiti university students. Doctor of Philosophy in Human Performance. Middle Tennessee State University. Published by ProQuest LLC.

5. Awadalla, N.J. Aboelyazed, A.E. Hassanein, M.A. Khalil, S.N. Aftab, R. Gaballa, I.I. And Mahfouz, A.A. (2014): Assessment of physical inactivity and perceived barriers to physical activity among health college students, south-western Saudi Arabia. Eastern Mediterranean Health Journal. 20 ( 10): 596-604.

6. Bray, S.R. (2007): Self-Efficacy for Coping with Barriers Helps Students Stay Physically Active during Transition to Their First Year at a University. Research Quarterly for Exercise \& Sport., 78, 61-70. http://dx.doi.org/10.1080/02701367.2007.10599404.

7. Dalibalta, S. \& Davison, G. (2016): Perceived Exercise Benefits and Barriers of a Mixed Student Population in the United Arab Emirates. International Journal of Scientific Research and Innovative Technology. (3), 23133759.

8. Daskapan, A., Tuzun, E.H. \& Eker, L. (2006): Perceived barriers to physical activity in university students. Journal of Sports Science and Medicine. (5): 615-620.

9. Global Recommendations on Physical Activity for Health: 18-64 years old [Internet]. Geneva: World Health Organization; 2011. Available at:

10. (http://www.who.int/dietphysicalactivity/physical-activityrecommendations-18-64years.pdf, accessed 2 July 2014).

11. Khalaf, A., Ekblom, O., Kowalski, J., Berggren, V., Westergren, A. \& Al-Hazzaa, H. (2013): Female university students' physical activity levels and associated factors: a cross-sectional study in Southwestern Saudi Arabia. Int J Environ Res Public Health. Aug; 10(8):3502-17. PMID:23939387

12. Lemos, R.I., Ribera, A.M. \& García, O.G. (2014): Perceived Barriers to Physical Activity and Related Factors in Spanish University Students. Open Journal of Preventive Medicine. (4), 164-174.

13. Lovell, G.P., El Ansari, W. and Parker, J.K. (2010): Perceived Exercise Benefits and Barriers of NonExercising Female University Students in the United Kingdom. International Journal of Environmental Research and Public Health. 7: 784-798.

14. http://dx.doi.org/10.3390/ijerph7030784

15. Munford, S.N. (2011): Gender differences in barriers to physical activity among college students reporting varying levels of regular physical activity. (Order No. 3468233, Walden University). ProQuest Dissertations and Theses, 186. Retrieved from https://ezproxy. mtsu.edu:3443/login?url=http://search.proquest.com/docview/887711895?accountid=488 6. (887711895).

16. Musaiger, A., Al-Kandari, F., Al-Mannai, M., Al-Faraj A., Bouriki F., Shehab F., et al. (2014): Perceived barriers to weight maintenance among university students in Kuwait: the role of gender and obesity. Environ Health Prev Med., 19(3):207-14.

17. Rajappan, R., Selvaganapathy, K. \& Liew, L. (2015): Physical activity level among university students: a cross sectional survey. Int J Physiother Res.; 3(6):1336-43.

18. Raynor, U \& Jankowiak, N. (2010): Accelerometry-determined adherence to the 2008 physical activity guidelines for Americans among college students. American Journal of Health Education. 41: 353-362.

19. Samara, A., Nistrup, A., Aro, A., \& Al-Rammah, T. (2015): Lack of facilities rather than sociocultural factors as the primary barrier to physical activity among female Saudi university students. International Journal of Women's Health. 7, 279-286. doi:10.2147/IJWH.S80680

20. Sultoni, J.K. \& Suherman, A. (2017): Barriers to Physical Activity on University Student. 1st Annual Applied Science and Engineering Conference.

21. World Health Organization (WHO) (2010): Reducing risks, promoting healthy life. The world health report. Available at: http://www. who.int/whr/2002/ chapter4/en/index4. html. Accessed September 2. 